\title{
The Forward Vertex upgrade detector for PHENIX
}

\author{
Sergey A. Butsyk ${ }^{\mathrm{a}}$ \\ for the PHENIX Collaboration \\ Los Alamos National Laboratory, MS H846 P-25 LANL, Los Alamos, NM 87545, USA
}

Received: 30 September 2008 / Revised: 10 March 2009 / Published online: 8 May 2009

(C) Springer-Verlag / Società Italiana di Fisica 2009

\begin{abstract}
The PHENIX detector at RHIC has been built with a strong heavy quark particles identification capability. These unique probes of matter are essential to adequately understand in-medium energy loss and to test the basic properties of QCD. The current PHENIX heavy flavor physics program will be significantly enhanced by the addition of the Forward Silicon Vertex upgrade detector (FVTX) in the acceptance of the existing muon arm detectors $(1.2<|y|$ $<2.4$ ). The proposed tracker is planned to be put into operation in FY2011. Each arm of the FVTX detector consists of 4 disks of silicon strip sensors combined with FPHX readout chips and provides a precision measurement of the radial coordinate of the track. The current status of the detector design and construction and expectations for the physics signal extraction will be presented.
\end{abstract}

PACS 25.75.-q $\cdot 29.40 . G x$

\section{Introduction}

The Forward Vertex Detector (FVTX) is an essential part of the PHENIX forward upgrade program. The full detector consists of two identical silicon vertex tracking arms, covering the acceptance of the existing muon spectrometers in the forward and backward rapidity range $(1.2<|y|<2.4)$. The FVTX detector is a part of the PHENIX silicon vertex upgrade and mechanically shares the enclosure with the barrel vertex tracker (see Fig. 1). Each FVTX detector arm consists of 4 disks of silicon strip detectors with $75 \mu \mathrm{m}$ wide strips, positioned along $\phi$-direction, giving a precise measurement of the radial component of the charged particle track. The disk closest to the interaction point is smaller in radius than the remaining three disks and each disk of the detector constructed as a set of 48 identical blocks, named "wedges". Each wedge covers an active area of $3.25^{\circ}$ in $\phi$

a e-mail: butsyk@ rcf.rhic.bnl.gov and consists of a silicon strip sensor, readout chips, and a kapton high-density interconnect to send the data out to the readout boards. The silicon sensor has a total of 1664 strips, which length is gradually increasing from $2.8 \mathrm{~mm}$ for the innermost radius up to $11.2 \mathrm{~mm}$ at the outermost radius of the large disk. The readout FPHX chip is designed at FNAL and reads the data from 128 strips, performs digitization and zero suppression of the data and sends the data out on 2 serial data lines. To read out the data from one "wedge" we need 26(10) readout chips for the large and small disk respectively. The active areas of the neighboring wedges have an overlap in $\phi$, so the detector provides full $\phi$ coverage. The full FVTX detector has 1.1 million active strip channels.

\section{Physics motivations}

The main purpose of the Forward vertex Detector construction is to provide a precise measurement of the track vertex for the tracks reconstructed by the PHENIX muon arms. The muon arms are designed specifically to measure the muons in the forward and backward rapidity regions by absorbing much of the hadronic yield in several layers of steel absorber. The track's momentum is measured by a set of 3 Muon Tracker detectors, placed in the area of a strong magnetic field. The muon identification is performed by five layers of Muon Identifier detector, which measure the track's coordinates while further suppressing hadronic background contributions. There is currently no precise vertex tracker in PHENIX, so all the registered particles are assumed to originate from the primary vertex, which is measured by the Beam-Beam Counter detector. The current accuracy of the production vertex measurement is therefore poor (several $\mathrm{mm}$ in $\mathrm{Au}+\mathrm{Au}, \sim 2 \mathrm{~cm}$ in $\mathrm{p}+\mathrm{p}$ collisions).

The main physics analysis via single muons is the measurement of open heavy quark (charm and bottom) production cross section through disentangling the muons that originated from semi-leptonic decays of those particles. The 
Fig. 1 Conceptual design view of the vertex upgrade detectors for PHENIX. $1 / 2$ of the FVTX arm is shown in more details

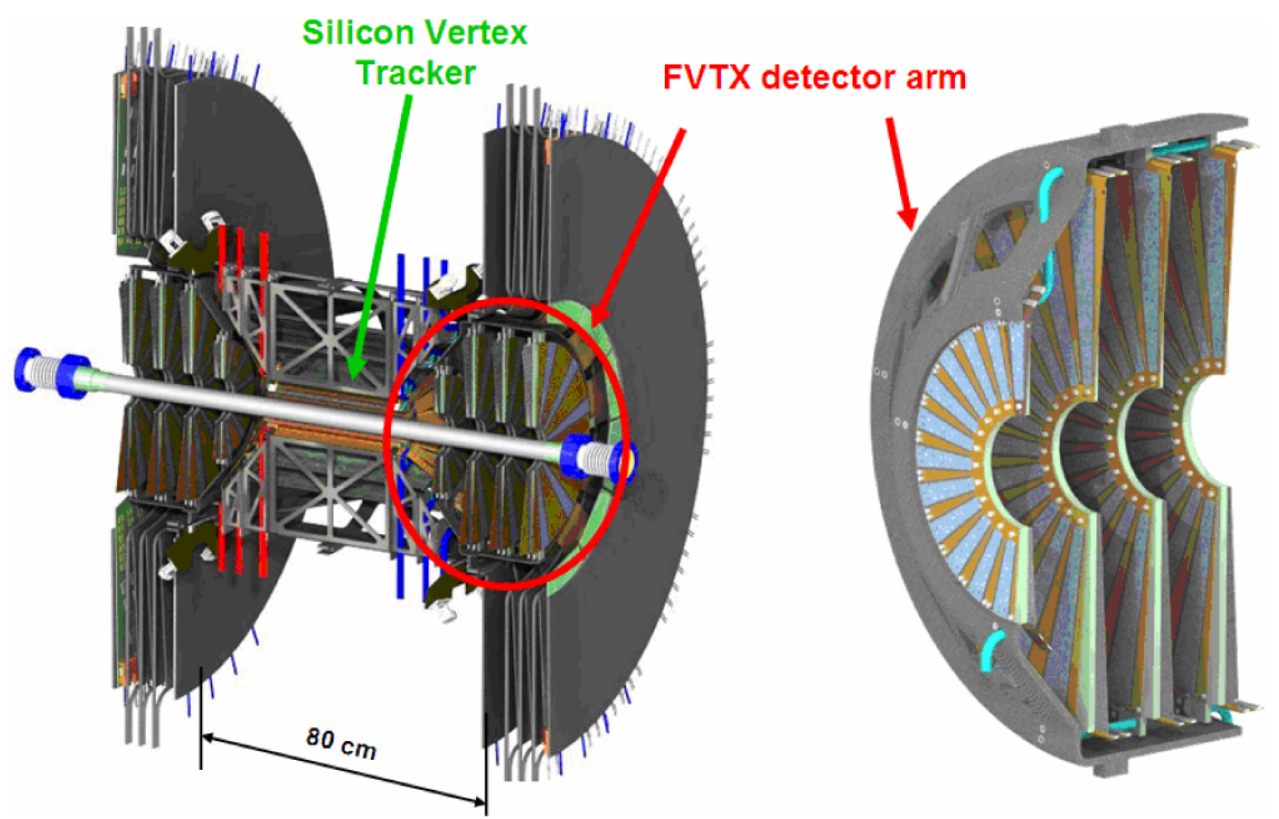

background for those measurements includes: a) prompt muons - muons originating from primary vertex; b) hadron decay muons; c) "punch-through" hadrons-hadrons that penetrate through the detector. Single muon analysis of the existing $\mathrm{p}+\mathrm{p}$ data has been successfully accomplished for Year-02 and Year-05 experimental Run data [1, 2]. The main source of the systematic errors in those analyses comes from the lack of accurate knowledge of the hadronic decay and "punch-through" background. The analysis requires understanding the yield of the hadrons at forward rapidity, and also requires a rigorous simulation of the propagation of those hadrons through the detector material in order to estimate the survival probability and decay kinematics. Overall the $\mathrm{S} / \mathrm{B}$ ratio for the heavy flavor muons is on the order of 0.1 to 0.5 at the momentum range of measurement. This results in a large systematic error for the heavy quark yields, making the interpretation of the analysis results challenging.

The FVTX upgrade is going to provide a precision measurement of the production vertex by calculating the distance of closest approach (DCA) of the muon track to the primary vertex (reconstructed by the barrel vertex tracker). The DCA resolution in radial direction is expected to be approximately $100 \mu \mathrm{m}$. The detector will add 4 spatial points close to the vertex to the muon arm detector hits, substantially increasing the precision of a combined multidimensional Kalman fit, used for the track reconstruction. Requiring the DCA to be less than some cut-off will effectively remove the major portion of the decay muons. On the other hand requiring the DCA to be larger than some cut-off can suppress the prompt muon contribution. The knowledge of DCA can also be used to disentangle the open charm and open bottom particle decay components in the muon spectrum.
The other important physics topic that is going to be improved significantly with the addition of the FVTX detector is the measurement the di-muon decays of the heavy quarks resonance states $\left(J / \psi, \psi^{\prime}, Y \rightarrow \mu^{+} \mu^{-}\right)$. Existent PHENIX measurements [3, 4] are going to be improved both in terms of the background rejection and due to improved momentum resolution (and therefore mass resolution).

\section{Simulation results}

In order to understand the detector capabilities of delivering the expected results, a full simulation study of the main physics observables has been performed. Figure 2 shows simulation results for the ratio of the signal to background for single muon analysis for D and B meson decays as a function of muon $p_{T}$. Different curves on the plots indicate the S/B without the FVTX (lower curves), and after successive FVTX cuts are applied. The main conclusion from this study is that with a set of cuts on DCA and $\chi^{2}$ of the fitted track we improve the S/B ratio by a factor of 10 over the whole momentum range. This factor can be translated into $\times 8$ improvement of the systematic uncertainties and $\times 3$ improvement of statistical errors. The total error that one expects for the extraction of the heavy flavor muon signal is shown in Fig. 3 in comparison with the error levels that we obtained from Year-02 analysis [1].

Simulation result for the $J / \psi, \psi^{\prime}$ signal reconstruction is shown in Fig. 4 for the case of $\mathrm{Au}+\mathrm{Au}$ events. It is clearly seen that the background level reduces to a comfortable level due to decay muon rejection and the mass resolution improves to the level that we start to clearly resolve $J / \psi$ and $\psi^{\prime}$ peaks from the mass spectrum. 
Fig. 2 Signal to background ratio for single muon analysis for D (left) and B (right) meson decays. Different curves show different FVTX cuts applied to the data

Fig. 3 Total error expectations for single muon component from heavy quarks in comparison with published results from Year- $02 p+p$ run results [1]

Fig. 4 Simulation of quarkonia states reconstruction in $\mathrm{Au}+\mathrm{Au}$ collisions with realistic background contributions without FVTX detector (left) and with the FVTX detector (right)
D- $->\mu-$
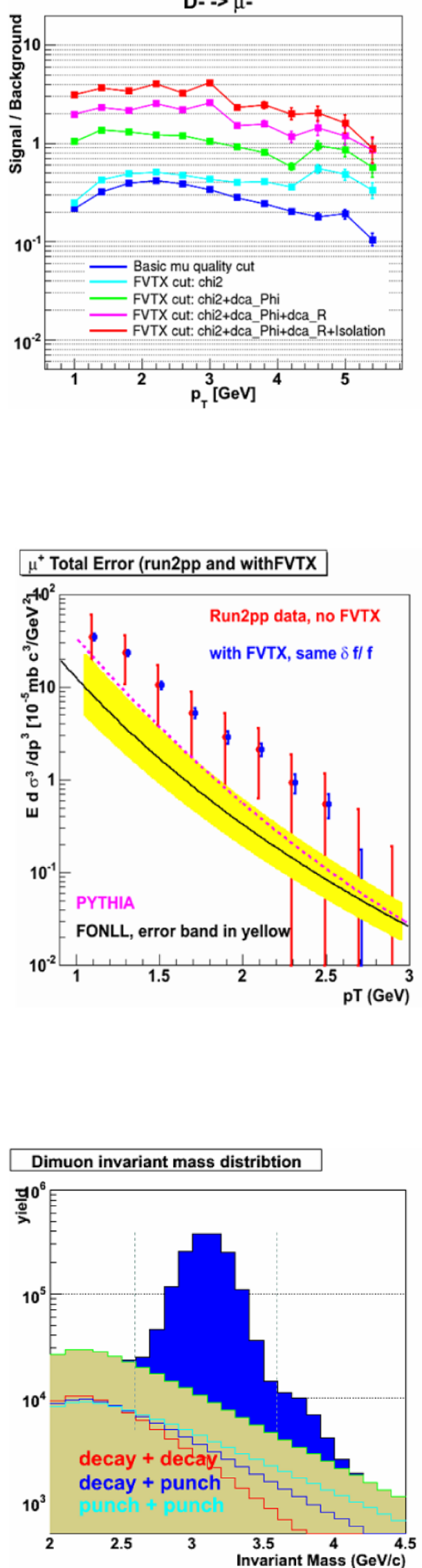

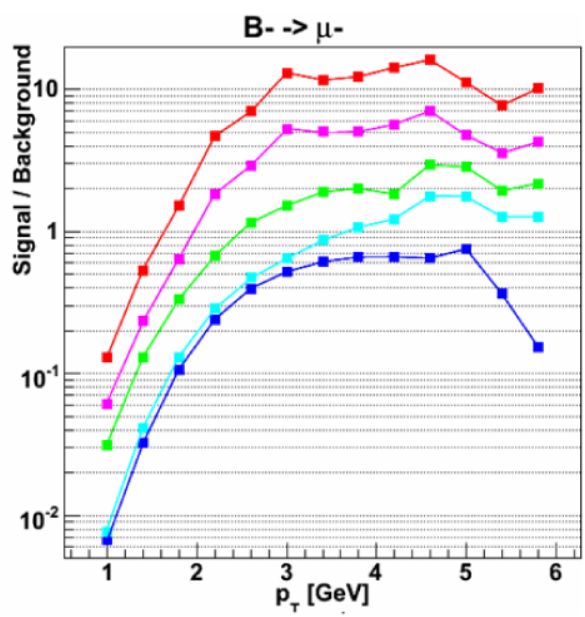

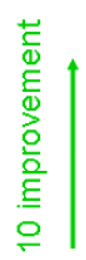
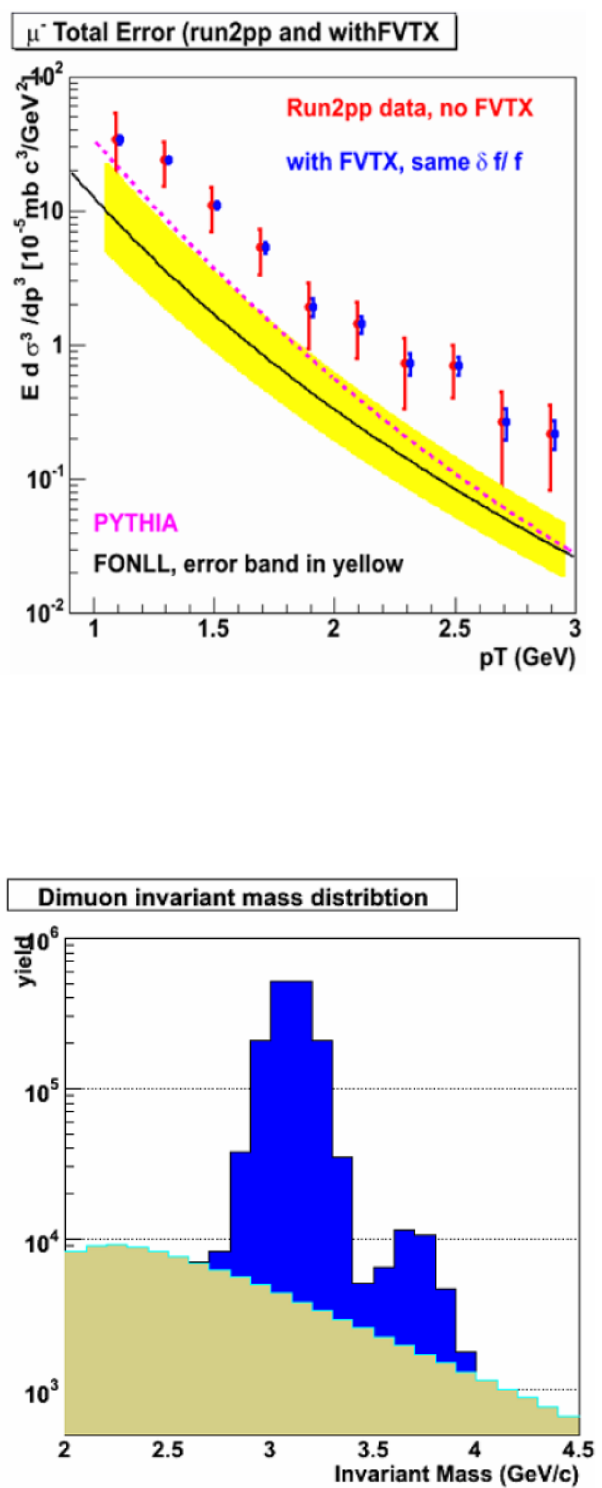
Simulations also showed that the FVTX detector will play important role in: a) double spin asymmetry $A_{L L}$ measurement, b) search for the muons originating from $\mathrm{W}$-boson decays, c) separation of charm and bottom through the DCA.

\section{Conclusions}

A large spectrum of physics analysis (including open charm and charmonium reconstruction, W-boson tagging and double-spin asymmetry measurements) in the forward and backward rapidity region becomes available for the first time or significantly improved with the addition of the FVTX detector. The precision knowledge of the muon production vertex will help to separate different sources of backgrounds, improve tracking efficiency and momentum resolution. The FVTX upgrade proposal has been approved for construction and funded by the Department of Energy in April 2008 and the detector will be put into operation in the 2011 experimental run.

\section{References}

1. S.S. Adler et al. (PHENIX Collaboration), Phys. Rev. D 76, 092002 (2007)

2. D. Hornback, in Quark Matter 08 Proceedings. arXiv:0804.4825v1 [nucl-ex]

3. A. Adare et al. (PHENIX Collaboration), Phys. Rev. C 77, 024912 (2008)

4. A. Adare et al. (PHENIX Collaboration), Phys. Rev. Lett. 98, 232002 (2007) 